\title{
Epidemiological and clinical aspects of pemphigus in Senegal
}

\section{Moussa Diallo, Boubacar Ahy Diatta, Aassane Diop, Maodo Ndiaye, Mame Téné Ndiaye, Birame Seck, Aminata Deh, Kadim Diop, Suzanne Niang}

Department of Dermatology, Aristide Ledantec University Hospital of Dakar, Senegal

Corresponding author: Prof. Moussa Diallo, E-mail: moussante@hotmail.com

\begin{abstract}
Background: The purpose of this study was to specify the epidemiological and clinical characteristics of pemphigus in the Department of Dermatology of the University Hospital of Dakar. Patients and Methodology: All medical records of patients seen for pemphigus in our department over a 11-year period were analyzed retrospectively. Results: We collected 105 cases of pemphigus, corresponding to a frequency of 10 cases/year and a hospital prevalence of $2.7 \%$. Thus, pemphigus held the second rank of blistering autoimmune bullous diseases after bullous pemphigoid. The mean age of patients was 47.62 years and the age group [40-59 years] was predominantly affected (28 cases; $45 \%)$. There were 41 femeles and 21 men (F/M ratio: 2). We did not find an endemic focus in our country. Clinical forms were dominated by pemphigus foliaceus (30 cases; $48.5 \%$ ), followed by pemphigus vulguaris (19 cases; $30.6 \%$ ) and finally by pemphigus erythematosus (13 cases; $21 \%)$. In 16 cases $(25 \%)$, the pemphigus had begun after the consumption of a traditional medicinal plant (8 cases). An associated toxidermia was found in 12 cases. Pruritus was present in 43 patients (69.35\%). Discussion: In Senegal, pemphigus are common diseases, but without however an endemic focuc. They occur in young women, with a predominance of superficial forms, especially pemphigus foliaceus. The presence of pruritus and associated toxidermia to traditional medicinal plants are common.
\end{abstract}

Key words: Pemphigus; Autoimmune Bullous Dermatoses; Acantholysis 


\title{
Aspects epidemiologiques et cliniques des pemphigus au Senegal
}

\section{Moussa Diallo, Boubacar Ahy Diatta, Aassane Diop, Maodo Ndiaye, Mame Téné Ndiaye, Birame Seck, Aminata Deh, Kadim Diop, Suzanne Niang}

\author{
Department of Dermatology, Aristide Ledantec University Hospital of Dakar, Senegal
}

Corresponding author: Prof. Moussa Diallo, E-mail: moussante@hotmail.com

\begin{abstract}
RÉSUMÉ
Introduction: Lobjectif de cette étude était de préciser les aspects épidémiologiques et cliniques des pemphigus au CHU de Dakar. Patients et Méthodes: Tous les dossiers des malades admis pour pemphigus ont été analysé de façon rétrospective sur une période de 11 ans. Résultats: Nous avons colligé 105 cas de pemphigus, correspondant à une fréquence de 10 cas/an et une prévalence hospitalière de $2,7 \%$. Les pemphigus occupaient le $2^{\circ}$ rang des dermatoses bulleuses auto-immunes après la pemphigoide bulleuse. Lâge moyen des patie nts était de 47,62 ans et la tranche d'âge [40-59] ans était la plus concernée (28 cas; $45 \%$ ). Il s'agissait de 41 femmes et 21 hommes, soit un sex-ratio F/H de 2. Il n'existait pas de foyer endémique. Les formes cliniques étaient dominées par le pemphigus foliacé (30 cas; $48,5 \%$ ), suivi du pemphigus vulguaire (19 cas; 30,6\%) et enfin du pemphigus séborrhéique (13 cas; 21\%). Dans 16 cas $(25 \%)$, le pemphigus avait débuté au décours d'une phytothérapie orale (8 cas). Une toxidermie surajoutée était retrouvée dans 12 cas. Le prurit était noté chez 43 patients (69,35\%). Discussion: Au Sénégal, les pemphigus sont des affections fréquentes, mais sans toutefois de foyer endémique et surviennent chez des femmes jeunes, avec une prédominance des pemphigus superficiels, notamment du pemphigus foliacé. La présence d'un prurit et d'une toxidermie surajoutée aux plantes traditionnelles sont fréquentes.
\end{abstract}

Mots clès: Pemphigus; Dermatoses Bulleuses Auto-immunes; Acantholyse

\section{INTRODUCTION}

Les pemphigus sont des affections fréquentes en pratique dermatologique. Ils se rencontrent à tout âge et dans tous les groupes ethniques. Cependant, leurs caractéristiques épidémiologiques et cliniques restent très variables selon les régions du monde et selon les groupes ethniques. Ainsi, le Pemphigus Foliacé (PF) demeure endémique en Amérique du Sud et en Tunusie, alors que le pemphigus herpétiforme est plus décrit en Europe de l'Est [1,2]. Cette disparité serait probablement liée à des facteurs génétiques et environnementaux. En Occident, Asie et en Amérique latine, les particularités des pemphigus ont été bien caractérisées [3-11]. Par contre, en Afrique noire, l'étude des pemphigus a été souvent limitée par la non disponibilité des examens complémentaires, surtout des l'immunofluorescences, indispensables au diagnostic[12-14]. Lobjectif de ce travail était de préciser les aspects épidémiologiques et cliniques des pemphigus dans le service de Dermatologie du CHU de Dakar.

\section{PATIENTS ET MÉTHODES}

Nous avons analysé de façon rétrospective tous les dossiers des malades admis pour pemphigus dans le service de Dermatologie du CHU de Dakar sur une période de 11 ans (2004-2014). Le diagnostic de pemphigus était basé sur les données cliniques, du cytodiagnostic de Tzanck, de l'histopathologie cutanée et de l'immunofluorescence indirecte.

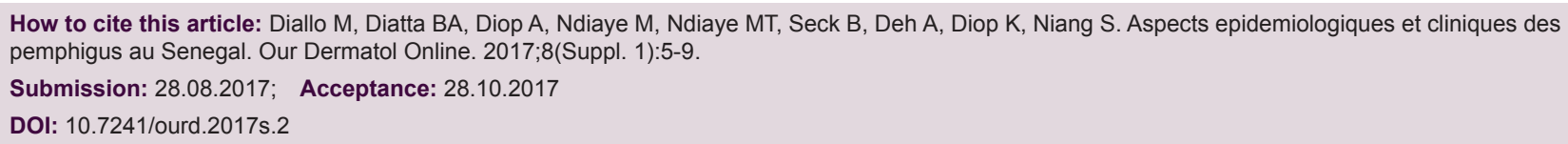




\section{RÉSULTATS}

Nous avons colligé 105 cas de pemphigus, correspondant à une fréquence de 10 cas/an et une prévalence hospitalière de $2,7 \%$. Durant la même période d'étude, les pemphigus occupaient le $2^{\circ}$ rang des dermatoses bulleuses auto-immunes dans notre service après la pemphigoide bulleuse. Cependant, seuls 62 dossiers avaient réuni les critères diagnostiques et avaient pu être analysés. Lâge moyen des patients était de 47,62 ans (extrêmes 5-90 ans) et la tranche d'âge (40-59) ans était la plus concernée (28 cas; 45,16\%) (Fig. 1). Lâge moyen était de 29 ans dans le Pemphigus Vulgaire (PV) et de 51 ans dans les pemphigus superficiels. Il s'agissait de 41 femmes et 21 hommes, soit un sex-ratio $\mathrm{F} / \mathrm{H}$ de 2. La prédominance féminine était observée dans toutes les formes cliniques, mais plus prononcé dans le Pemphigus Séborrhéique (PS). Nous n'avons pas trouvé de prédominance ethnique de la maladie, dont la répartition était superposable à celle de la population générale. De même, il n'existait pas de prédisposition liée à une origine géographique, ni à une profession, ni à un niveau socio-économique quelconque des patients. Il n'existait pas de cas de pemphigus familiaux. Les formes cliniques étaient dominées par le PF (30 cas; $48,5 \%$ ), suivi du PV (19 cas; $30,6 \%$ ) et enfin du PS (13 cas; $21 \%$ ) (Figs. 2 et 3). Le délai moyen de consultation était de 7,76 mois (extrêmes 6 j et 8 mois). Dans 16 cas $(25 \%)$, le pemphigus avait débuté au décours d'une phytothérapie orale. A l'admission, les patients présentaient tous des bulles flasques, avec signe de Nikolsky, associées dans 12 cas, à des lésions bulleuses nécrotiques, évocatrices de toxidermie surajoutée (Fig. 4). Dans toutes les formes de pemphigus, le prurit était présent chez 43 cas $(69,35 \%)$. Dans le PV, il existait une atteinte unguéale (8 cas) et une atteinte des muqueuses buccale (15 cas), conjonctivale ( 5 cas), génitale (7 cas), œsophagienne (lcas) et anale (lcas). Des complications à type d'infection herpétique (15 cas) et bactérienne (21 cas) étaient notées. Le cytodiagnostic de Tzanck était contributif dans 36 (58\%), alors que l'examen histopathologique était celui d'un pemphigus chez tous les malades inclus dans l'étude. De même, tous les patients présentaient des anticorps anti-substances intercellulaires circulants. Limmunofluorescence directe n'était pas disponible. Tous les malades étaient traités par corticothérapie en bolus ( 1 cas) ou par voie orale $(61$ cas) entre 0,5 et $2 \mathrm{mg} / \mathrm{kg} / \mathrm{j}$, associée à la dapsone (3 cas), azathioprime ( 4 cas) et au méthotrexate ( 1 cas). Le décès, noté dans

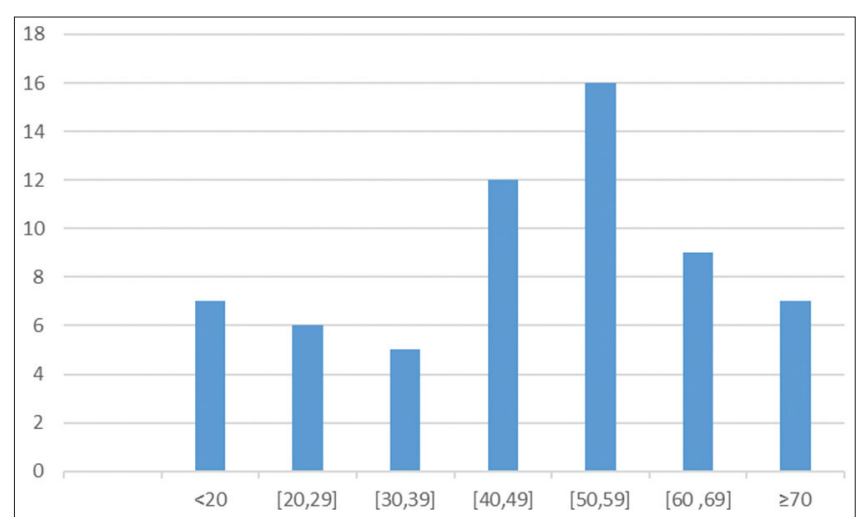

Figure 1 : Répartition des pemphigus selon l'âge.

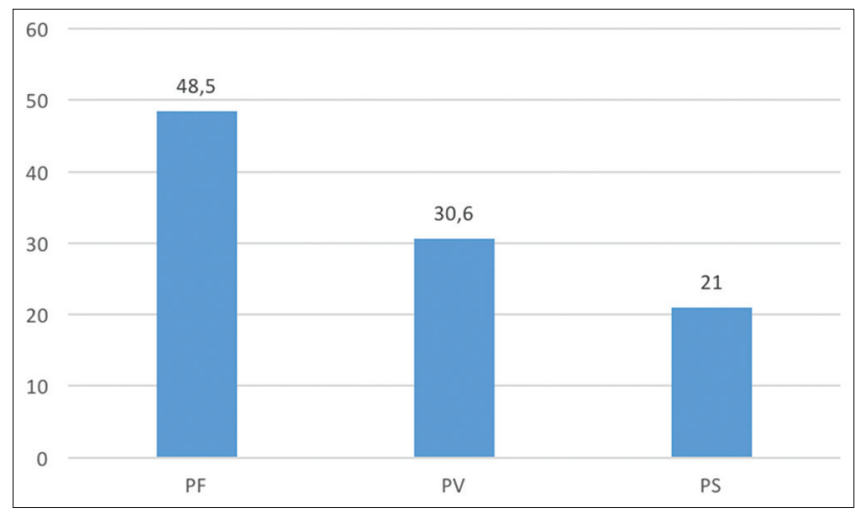

Figure 2: Fréquence (en \%) des différentes formes cliniques de pemphigus.

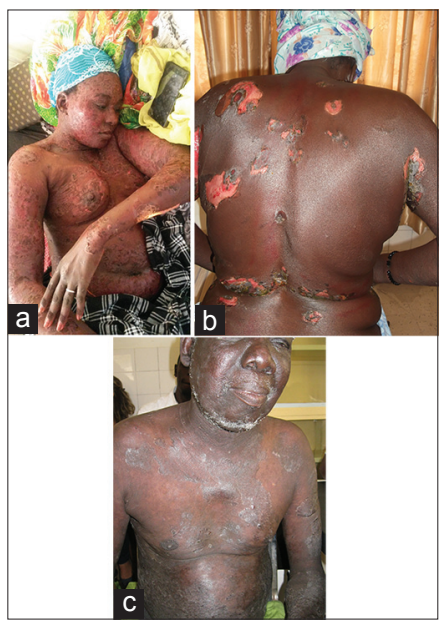

Figure 3: Pemphigus foliacé (a), pemphigus vulgaire (b) et pemphigus séborrhéique (c).

6 cas $(9,6 \%)$ était secondaire à une infection ( 5 cas) et d'origine non précisé (l cas). Une rechute était observée chez 23 patients (37\%), suite le plus souvent à une rupture thérapeutique intempestive ( 12 cas) ou à une surinfection herpétique (8), une phytothérapie (1 cas) ou à la dégression de la corticothérapie (2 cas). 


\section{DISCUSSION}

Les pemphigus sont des affections fréquemment rencontrées en Afrique, occupant ainsi le $2^{\circ}$ rang des dermatoses bulleuses auto-immunes dans notre service après la pemphigoide bulleuse. La fréquence du pemphigus observée dans notre étude (10 cas/an) était comparable à celle rapportée en Afrique du Sud (9,33 cas/an), mais plus faible qu'en Tunisie (18,4cas/ an) et au Brésil (12,66 cas/an) [12,15,16]. Cette différence pourrait s'expliquer par l'existence de formes endémiques dans ces 2 régions.

Comme dans la plupart des autres pays en Afrique noire, les pemphigus se rencontrent en majorité chez des adultes jeunes entre 40 et 60 ans [12-14]. Lâge de survenue semble plus précoce dans le PV (29 ans) que dans les pemphigus superficielles ( 51 ans). Par contre, le pemphigus survient plus précocément en Tunisie (28 ans) et au Brésil (25 ans), et plus tardivement en Europe (50-70 ans), avec un âge moyen de 62 ans en Allemagne $[6,15,16]$.

La prédominance féminine notée dans notre étude a été aussi retrouvée dans toutes les autres séries

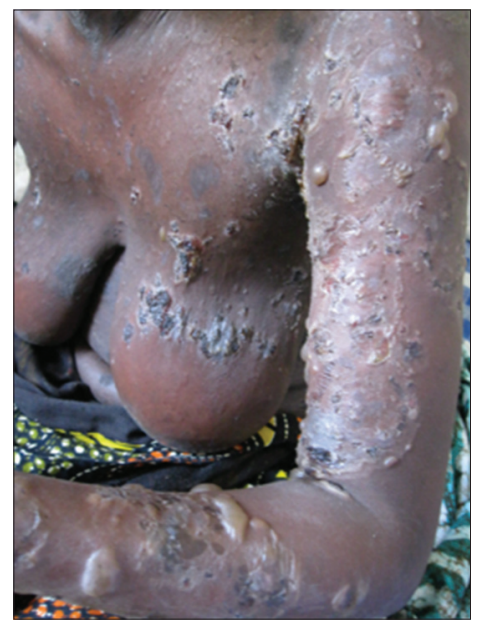

Figure 4: Lésions nécrotiques évocatrices de toxidermie surajoutée au cours d'un pemphigus vulgaire. africaines, mais de façon plus marquée au Mali et en Tunusie $[13,15]$. Ceci pourrait probablement être également lié à la fréquence élevée des PF qui prédominent largement chez la femme [15]. Par contre, au Moyen-Orient, le pemphigus semble prédominait chez l'homme [8].

Dans notre étude, nous n'avons pas trouvé de prédominance ethnique du pemphigus, contrairement au Mali où il était préférentiellement observé chez des peuls [13]. De même, il n'existait pas de prédisposition liée à l'origine géographique, ni à la profession, ni au niveau socio-économique des patients. Ces résultats témoignent de l'absence de foyer endémique au Sénégal, contrairement en Tunusie et au Brésil $[15,16]$.

Contrairement en Asie, au Moyen-Orient et en Europe où le PV est de loin le plus fréquent, notre étude confirme la prédominance en Afrique des pemphigus superficiels (69,5\%), notmamment du PF $(48,5 \%)$ [3-5,7-9,12,13] (Tableau 1). La plus grande fréquence du PF comparée au PS a été aussi notée en Afrique du Sud, Tunusie et Mali $[12,13,15]$.

Le délai diagnostic était tardif, en moyenne de 7,5 mois, probablement lié au déficit en spécialistes, mais aussi à la méconnaissance des formes débutantes. Ce retard diagnostique explique la fréquence des complications infectieuses surtout herpétiques, dans notre série.

Lévolution chronique des pemphigus et la connation souvent mystique des maladies dermatologiques en Afrique expliquent le recours fréquent aux plantes traditionelles.

Chez 16 de nos patients (25\%), l'éruption bulleuse a été déclenchée au décours d'une phytothérapie orale. En effet, ces plantes traditionnelles pourraient contenir des dérivés thiolés dont le rôle inducteur est bien connu [17]. Ceci pourrait aussi expliqué que certains patients présentaient en plus des bulles classiques de

Tableau 1 : Tableau comparatif des formes cliniques de pemphigus selon les pays

\begin{tabular}{|c|c|c|c|c|}
\hline Frequence (\%) & P. foliacé & P.séborrhéique & P. vulgaire & P.végétant \\
\hline Sénégal & 48 & 21 & 29 & 0 \\
\hline Tunisie [15] & 83.33 & 0 & 8.33 & 4.16 \\
\hline Afrique du sud [12] & 55.36 & 0 & 44.64 & 0 \\
\hline Mali [13] & 83 & 0 & 13.33 & 3.33 \\
\hline Turquie [4] & 6.9 & 0 & 93.1 & 0 \\
\hline Taïwan [9] & $35.19 \%$ & $0 \%$ & $62.7 \%$ & $0 \%$ \\
\hline Midi-Pyrénées [5] & $20.4 \%$ & $7.4 \%$ & $72.2 \%$ & $0 \%$ \\
\hline Espagne [3] & 13 & 4 & 79 & 4 \\
\hline
\end{tabular}


pemphigus, des lésions nécrotiques évocatrices de toxidermie surajoutée.

Le prurit est en principe absent ou très rare dans le pemphigus. Dans notre série, le pemphigus était remarquable par son caractère fréquemment prurigineux (70\%), comme rapportée dans des études antérieures en Afrique noire [12-14]. Cependant l'aspect en hypopion fréquemment décrit au Mali et en Afrique du Sud n'avait pas été noté chez nos malades [12,13].

Le bas niveau socio-économique des patients, la connotation souvent mystique des dermatoses, l'évolution chronique de ces affections, expliquent la fréquence des rechutes souvent liées à un arrêt thérapeutique intempestif.

Ces rechutes très courantes, ainsi que les fréquentes complications de la corticothérapie pourraient être significativement dimuniées grâce au rituximab, mais non accessible du fait de son coût encore élevé.

\section{CONCLUSION}

Notre étude confirme les résultats des rares études antérieures en Afrique noire sur les pemphigus. Il s'agit d'affections fréquentes, mais sans toutefois de foyer endémique, survenant chez des femmes jeunes, avec une prédominance des pemphigus superficiels, notamment du pemphigus foliacé. La présence d'un prurit et d'une toxidermie surajoutée aux plantes traditionnelles sont fréquentes. Lamélioration des moyens diagnostiques pourrait permettre une meilleure caractérisation de ces affections en Afrique noire, à travers des études multicentriques. Leur pronostic pourrait également être amélioré par l'accés au rituximab.

\section{RÉFÉRENCES}

1. Alpsoy E, Akman-Karakas A, Uzun S. Geographic variations in epidemiology of two autoimmune bullous diseases: pemphigus and bullous pemphigoid. Arch Dermatol Res. 2015;307:291-8.
2. Laws PM, Heelan K, Al-Mohammedi F, Walsh S, Shear NH. Pemphigus herpetiformis: a case series and review of the literature. Int J Dermatol. 2015;54:1014-22.

3. Meyer N, Misery L. Geoepidemiologic considerations of autoimmune pemphigus. Autoimmun Rev. 2010;9:A379-82.

4. Saleh MA. Pemphigus in the Arab world. J Dermatol. 2015;42:27-30.

5. Thomas M, Paul C, Berard E, Fortenfant F, Mazereeuw-Hautier J, Livideanu $\mathrm{C}$, et al. Incidence of auto-immune pemphigus in the Midi-Pyrénées region in 2002-2006. Dermatology. 2010;220:97-102.

6. Bertram F, Bröcker EB, Zillikens D, Schmidt E. Prospective analysis of the incidence of autoimmune bullous disorders in Lower Franconia, Germany. J Dtsch Dermatol Ges. 2009;7:434-40.

7. Langan SM, Smeeth L, Hubbard R, Fleming KM, Smith CJ, West J. Bullous pemphigoid and pemphigus vulgaris--incidence and mortality in the UK: population based cohort study. BMJ. 2008;9;337:a180.

8. Jowkar F, Sadati MS, Tavana S, Agah MA. Epidemiology of autoimmune bullous diseases and therapeutic modalities during a 10 year period in Iran. Acta Dermatovenerol Croat. 2014;22:246-9.

9. Huang YH, Kuo CF, Chen YH, Yang YW. Incidence, mortality, and causes of death of patients with pemphigus in Taiwan: a nationwide population-based study. J Invest Dermatol. 2012;132:92-7.

10. Ishii N, Maeyama Y, Karashima T, Nakama T, Kusuhara M, Yasumoto S, et al. A clinical study of patients with pemphigus vulgaris and pemphigus foliaceous: an 11-year retrospective study (1996-2006). Clin Exp Dermatol. 2008;33:641-3.

11. Gonçalves GA, Brito MM, Salathiel AM, Ferraz TS, Alves D, Roselino AM. Incidence of pemphigus vulgaris exceeds that of pemphigus foliaceus in a region where pemphigus foliaceus is endemic: analysis of a 21-year historical series. An Bras Dermatol. 2011;86:1109-12.

12. Aboobaker J, Morar N, Ramdial PK, Hammond MG. Pemphigus in South Africa. Int J Dermato. 2001;40:115-9.

13. Mahé A, Flageul B, Cissé I, Kéita S, Bobin P. Pemphigus in Mali: a study of 30 cases. Br J Dermatol. 1996;134:114-9.

14. Ndiaye A, Strobel M, Ball M, Marchand JP, Ndiaye B. [Pemphigus in Sénégal(a propos of 40 cases)]. Dakar Med. 1982; 27(1):97-102.

15. Zaraa I, Kerkeni N, Ishak F, Zribi H, El Euch D, Mokni M, et al. Spectrum of autoimmune blistering dermatoses in Tunisia: an 11-year study and a review of the literature. Int J Dermatol. 2011;50:939-44.

16. Pires CA, Viana VB, Araújo FC, Müller SF, Oliveira MS, Carneiro FR. Evaluation of cases of pemphigus vulgaris and pemphigus foliaceus from a reference service in Pará state, Brazil. An Bras Dermatol. 2014;89:556-61.

17. Brenner S, Goldberg I. Drug-induced pemphigus. Clin Dermatol. 2011;29:455-7.

Copyright by Moussa Diallo, et al. This is an open-access article distributed under the terms of the Creative Commons Attribution License, which permits unrestricted use, distribution, and reproduction in any medium, provided the original author and source are credited.

Source of Support: Nil, Conflict of Interest: None declared. 